\title{
Wnt Signaling in the Vertebrate Central Nervous System: From Axon Guidance to Synaptic Function
}

\author{
Patricia C. Salinas \\ Department of Cell and Developmental Biology, University College London, London WC1E 6BT, \\ United Kingdom \\ Correspondence: p.salinas@ucl.ac.uk
}

Regulation of cell signaling by Wnt proteins is critical for the formation of neuronal circuits. Wnts modulate axon pathfinding, dendritic development, and synaptic assembly. Through different receptors, Wnts activate diverse signaling pathways that lead to local changes on the cytoskeleton or global cellular changes involving nuclear function. Recently, a link between neuronal activity, essential for the formation and refinement of neuronal connections, and Wnt signaling has been uncovered. Indeed, neuronal activity regulates the release of Wnt and the localization of their receptors. Wnts mediate synaptic structural changes induced by neuronal activity or experience. New emerging evidence suggests that dysfunction in Wnt signaling contributes to neurological disorders. In this article, the attention is focused on the function of Wnt signaling in the formation of neuronal circuits in the vertebrate central nervous system.

$T^{\text {he }}$ he formation of neuronal connections requires the navigation of axons to their appropriate synaptic targets, the formation of terminal branches, and the assembly of functional synapses. These processes greatly depend on the proper dialogue between axons and their environment as they navigate to their target, and between axons and their postsynaptic dendrites during synapse assembly. A combination of secreted molecules and transmembrane proteins modulates these processes. Studies over the last 10 years have revealed an essential role for Wnt signaling in axon pathfinding, dendritic development, and synapse assembly in both central and peripheral nervous systems. Wnts also modulate basal synaptic transmission and the structural and functional plasticity of synapses in the central nervous system. Studies of Wnts in the nervous system have significantly contributed to our current understanding of the molecular mechanisms that control neuronal circuit assembly. These studies have also shed light into fundamental aspects of cell signaling such as novel mechanisms of protein secretion (Korkut et al. 2009) and receptor dynamics (Sahores et al. 2010). Here I review the mechanisms by which Wnts modulate axon guidance and synapse formation in the vertebrate central nervous system. I also discuss the increasing evidence in support for a role of Wnts in basal

Editors: Roel Nusse, Xi He, and Renee van Amerongen

Additional Perspectives on Wnt Signaling available at www.cshperspectives.org

Copyright (C) 2012 Cold Spring Harbor Laboratory Press; all rights reserved; doi: 10.1101/cshperspect.a008003

Cite this article as Cold Spring Harb Perspect Biol 2012;4:a008003 
P.C. Salinas

synaptic transmission, synaptic plasticity, and neurological disorders.

\section{AXON NAVIGATION AND TERMINAL REMODELING}

Wnt signaling controls neuronal polarity, promotes axon outgrowth, and regulates the navigation of axons to their final synaptic targets. The first clear evidence that Wnts regulate axon guidance came from studies using loss-of-function approaches in different development systems including vertebrates (Salinas and Zou 2008; Sanchez-Camacho and Bovolenta 2009; Fradkin et al. 2010). Wnts induce a range of responses including repulsion, attraction, and promoting axon branching.

\section{Axon Guidance}

In the vertebrate nervous system, Wnts also regulate the direction of axon growth and guidance. In the spinal cord, commissural axons turn anteriorly after crossing the midline following a posterior-to-anterior gradient of attractive cues. Indeed, Wnt 4 and Wnt7b form a gradient (anterior high-posterior low) in the floor plate. Gain of function of Wnt4 using cultured openbook explants from the spinal cord reveals that Wnt 4 is an attractive cue for commissural axons after midline crossing (Fig. 1) (Lyuksyutova et al. 2003). This response seems to be mediated by the Frizzled-3 (Fz3) receptor, because $F z 3$ mutant mice show anterior-posterior guidance defects after commissural axons have crossed the midline (Lyuksyutova et al. 2003).

In contrast to the attractive activity of Wnt4, other Wnts function as repulsive signals. Wnt1 and Wnt5a form a high-anterior-to-low-posterior gradient along the dorsal spinal cord and repel corticospinal axons that descend along the spinal cord (Liu et al. 2005). During the initial part of their trajectory, these axons are insensitive to the repulsive activity of Wnts owing to the lack of expression of the Ryk receptor. After crossing the midline and entering the rostral part of the spinal cord, these axons begin to express Ryk. Importantly, injection of antibodies against Ryk affects the posterior growth of cor- ticospinal axons (Liu et al. 2005). Together, these results strongly suggest that two opposing gradients guide axons in the spinal cord. Early in development, a ventral gradient of Wnt4 attracts commissural axons in a posterior-to-anterior direction. Postnatally, corticospinal axons, which descend along the dorsal funiculus, experience a repulsing gradient of Wnt 1 and Wnt5a as they grow down the spinal cord (Fig. 1C).

How does Wnt5a regulate the behavior of commissural axons? Wnt5a activates a noncanonical Wnt signaling pathway that requires Fz3 and the four-pass transmembrane protein Van Gogh/strabismus (Vang) and Jun-N-terminal kinase (JNK) to promote axon outgrowth (Shafer et al. 2011). Analyses in cell lines led to the surprising finding that the core scaffold protein Dishevelled-1 (Dvl1) antagonizes Vang function. Indeed, expression of Dvll decreases the ability of Wnt5a to activate JNK by increasing Fz3 phosphorylation and decreasing its internalization. Because receptor internalization is crucial for signaling, Dvl1 blocks Wnt5a signaling. In contrast, expression of Vang2 decreases Fz3 phosphorylation and promotes its internalization (Shafer et al. 2011). Thus, Vang2 and Dvll induce opposing effects on Fz3 localization. Although the effect of Dvll on the behavior of commissural axons has not been examined, the localization of Vang2 and Fz3 suggests that Vang2 induces the internalization of Fz3 and activation of the PCP pathway by antagonizing Dvll function. It would be of interest to know whether Dvll also plays a negative role in Wnt signaling in other cellular processes.

Activation of a non-canonical pathway through the Ryk receptor regulates axon guidance. In the corpus callosum, Ryk mutant cortical axons are able to cross the midline, but they show aberrant trajectories on the contralateral side. Wnt5a, which is expressed around the area of the corpus callosum, repels Ryk-expressing axons in cortical explants (Fig. 1A,B) (Keeble et al. 2006). Knockdown of Ryk decreases calcium transients and increases misrouting of post-crossing axons (Hutchins et al. 2011). Interestingly, blockade of IP3 receptors and TRP channels reduces the rate of outgrowth, but only inhibition of TRP induces guidance 
A

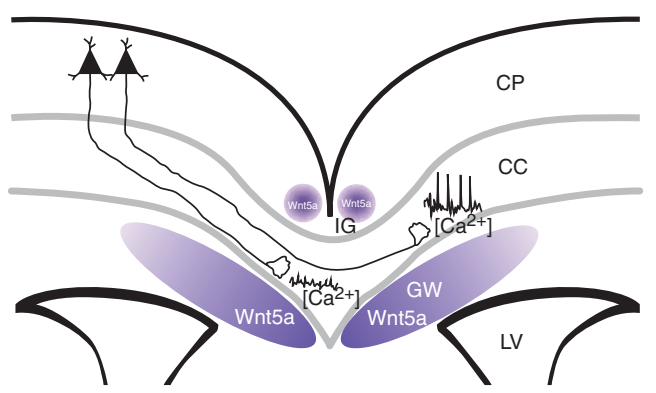

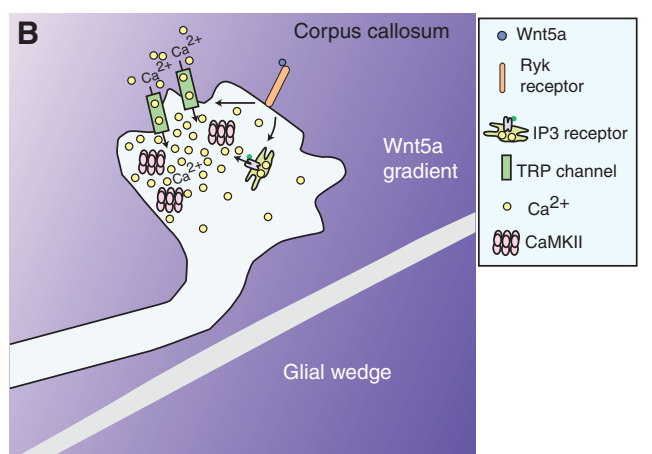

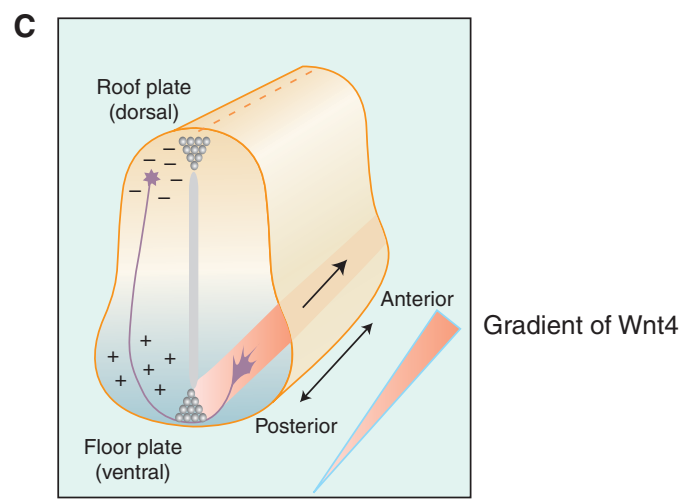

Figure 1. Gradients of Wnts regulate axon guidance in the spinal cord. $(A, B)$ In the cortex, a repulsive Wnt5 expressed in indusium griseum (IG) and in the glial wedge (GW) guides cortical axons through the corpus callosum (cc). Pyramidal cortical axons project to the contralateral side of the cortex by crossing the corpus callosum. After crossing to the contralateral side, these axons are repelled by Wnt5a, possibly by the expression of Ryk receptor in post-crossing axons. Wnt5a through Ryk induces the opening of IP3 and TRP channels to transiently activate CaMKII, resulting in increased axon outgrowth and repulsion. $(C)$ A gradient of Wnt4 in the floor plate controls the direction of commissural axons. After midline crossing, commissural axons turn in an anterior direction following the attracting gradient of Wnt4. (Panels $A$ and $B$ are from Hutchins and Kalil 2011; reprinted, with permission, from John Wiley \& Sons (C) 2011. Panel $C$ is from Imondi and Thomas 2003; reprinted, with permission, from the author.)

defects. Moreover, expression of a specific CaMKII inhibitory protein induces outgrowth and pathfinding defects (Hutchins et al. 2011). These results are consistent with previous studies showing that Wnt5a/Ryk signaling through CaMKII regulates axon outgrowth and turning of dissociated cortical neurons ( $\mathrm{Li}$ et al. 2009). Together, these results show that activation of Ryk receptors by different Wnts can trigger different axon guidance responses.

Although a role for Wnts in axon guidance has been shown in few brain areas, the broad but specific expression of different Wnts and their receptors in the nervous system suggests that these molecules play a general role in axon guidance. Studies addressing the contribution of Wnts together with other axon guidance molecules will be invaluable in understanding how axon behavior is controlled in normal and regenerative conditions.

\section{Formation of Topographical Maps}

Wnts also contribute to the formation of topographical maps in the brain. In the visual system, retinal ganglion cells (RGCs), organized in a two-dimensional (2D) layer, project their axons through the optic nerve into the brain 


\section{P.C. Salinas}

in an orderly manner. In the chick, axons from the dorsal retina project to the lateral part of the tectum, whereas axons from the ventral retina project to the more medial tectum. This retinotopic map permits an object projected into the retina to be properly represented in the tectum. Molecules such as Eph and Ephrin guide RGC axons to their appropriate terminal zone in the tectum (McLaughlin and O'Leary 2005). EphrinA molecules have been implicated in the formation of an anterior-posterior topographic map through their repulsive activity. In addition, a medial-to-lateral gradient of EphB in the retina with a medial-lateral gradient of Eph-
rinB1 in the tectum controls patterning through attraction (Hindges et al. 2002). Theoretical models propose that molecular gradients with opposing activities are required for the formation of this topographical map. Indeed, Wnts contribute to the formation of this retinotopic map. In the tectum, Wnt3 is expressed in a gradient from medial high to lateral low as observed for EphrinB1 (Fig. 2). In the retina, the Wnt3 receptor Ryk is expressed in a gradient from ventral high to dorsal low following the same pattern of EphB (Schmitt et al. 2006). In contrast to the attraction elicited by EphB and EphrinB1, Wnt3-Ryk induces repulsion (Schmitt et al.

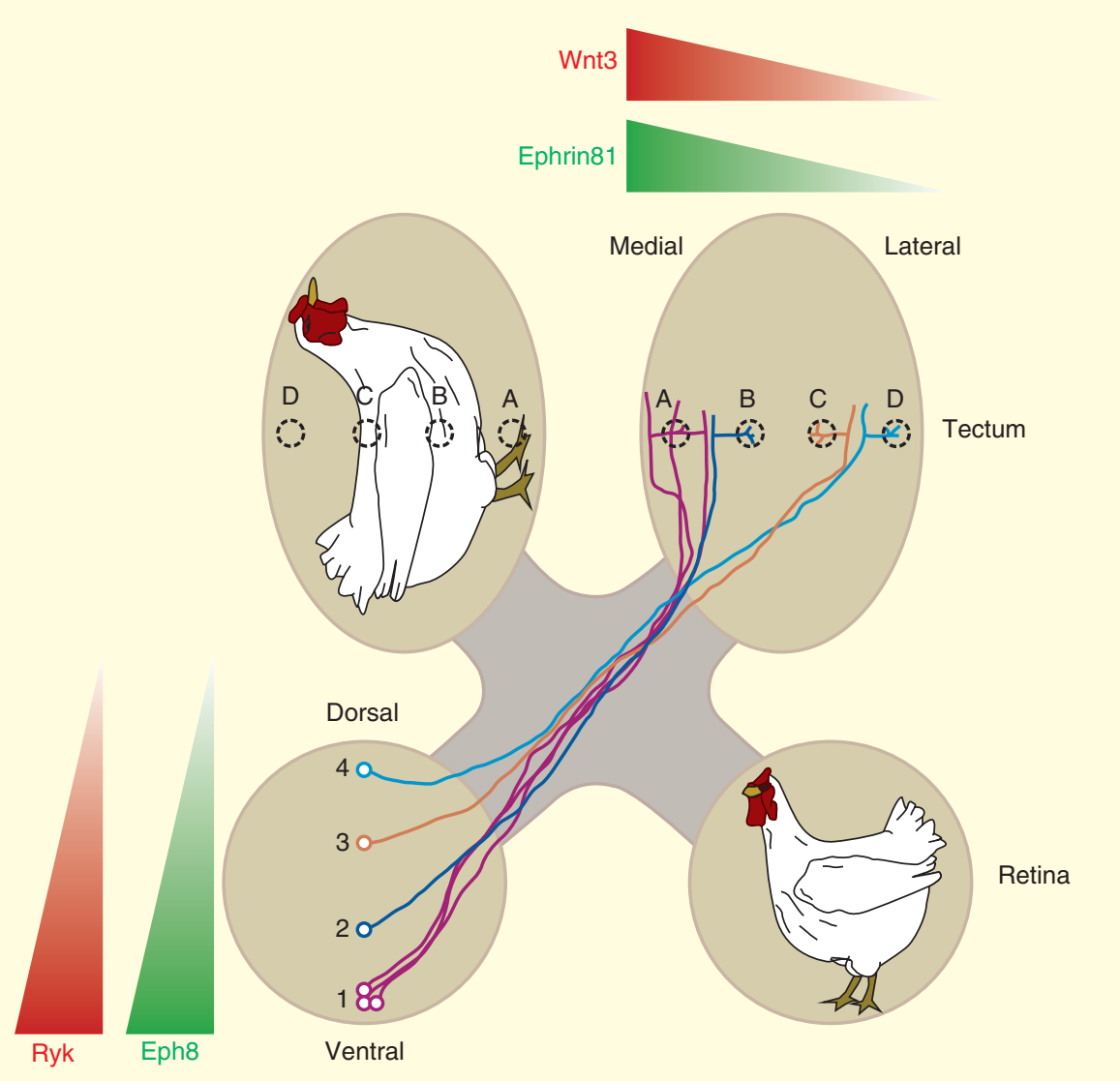

Figure 2. Wnt3-Ryk signaling controls the formation of topographical maps. In the retina, retinal ganglia cell axons express the Ryk receptor in a gradient from ventral high to dorsal low in the same manner as EphB. These axons project to the contralateral side and into the tectum whether they follow two opposing forces. Both Wnt3 and EphrinB1 are expressed in a gradient from medial high to lateral low. EphrinB-EphB1 forms an attractive medial-directing activity. In contrast, Wnt3-Ryk forms an opposing lateral-directing activity. (Figure is from Luo 2006; reprinted, with permission, from Nature (C) 2006.) 
2006). Thus, an attracting gradient of EphBEphrinB1 and an opposing repulsing gradient of Ryk-Wnt3 regulate the formation of the retinotopic map. The graded distribution of Wnts and their receptors in different brain regions strongly suggests that Wnts will also contribute to the formation of topographical maps in other sensory systems such as somatosensory and auditory systems.

\section{Axon Terminal Remodeling}

Wnt signaling promotes the terminal remodeling of axons before synapses begin to assemble. In cerebellar mossy fiber axons and DRG neurons, activation of the canonical Wnt pathway induces growth cone pausing, axon branching, and extensive enlargement of growth cones (Hall et al. 2000; Krylova et al. 2002). This behavior is distinct from the typical attractive or repulsive responses induced by axon guidance molecules. In contrast, this terminal remodeling is consistent with the response of axons as they arrive at their target fields and begin to form synapses. Studies in cultured neurons and at the Drosophila NMJ have shown that Wnt signaling through Dvl inhibits Gsk3 by a divergent canonical Wnt pathway that does not require transcription (Packard et al. 2002; Miech et al. 2008; Purro et al. 2008). In fact, terminal remodeling is achieved through profound changes on the axonal cytoskeleton. Wnt increases microtubule stability and induces the formation of microtubule loops within the growth cone, which contribute to growth cone pausing and enlargement (Ciani et al. 2004). Time-lapse analyses of the behavior of microtubule plus ends labeled with EB3-GFP reveal that Wnt3a induces the loss of microtubule growth direction, resulting in the formation of looped microtubules within the growth cone (Purro et al. 2008). This profound change in microtubule organization leads to growth cone pausing and enlargement. Two findings support a role for APC, a Wnt canonical component and a microtubule binding protein, in mediating growth cone remodeling. First, Wnt3a decreases the level of endogenous APC at the plus end of microtubules. Second, ShRNA knockdown of APC induces microtu- bule looping and axon remodeling, thus mimicking the effect of Wnt3a (Purro et al. 2008). Thus, Wnts regulate the organization of microtubules resulting in the terminal remodeling of growth cones, a crucial process in the conversion of actively growing growth cones into synaptic boutons.

\section{SYNAPSE FORMATION}

Synapse formation requires the proper apposition between the presynaptic terminal, containing the machinery necessary for neurotransmitter release, and the membrane of the postsynaptic cells containing neurotransmitter receptors and signaling molecules. Time-lapse studies have shown that presynaptic release sites form first, and they are followed by the accumulation of postsynaptic markers (Okabe et al. 2001). Although presynaptic release sites can assemble without clear evidence of postsynaptic assembly, these "orphan" sites are not stable. Time-lapse studies of dendritic spines, mushroom-like protrusions that receive excitatory inputs, revealed that these postsynaptic structures are very dynamic (Trachtenberg et al. 2002; Dunaevsky and Mason 2003), but their stability depends on their apposition to presynaptic boutons (Yoshihara et al. 2009). Thus, synaptic assembly requires the proper dialogue between the presynaptic terminal or bouton and its postsynaptic target cells. Several studies now show that bidirectional Wnt signaling contributes to this trans-synaptic dialogue.

\section{Presynaptic Assembly}

Studies using cultured neurons and analyses of Wnt-null mutant mice have led to the discovery that Wnts promote synapse formation. Wnt7a is expressed in cerebellar granule cells as they begin to contact their presynaptic mossy fiber axons (Hall et al. 2000). The mossy fiber-granule cell synapse is characterized by the interdigitation of several granule cell dendrites into a single mossy fiber axon, resulting in the formation of a complex synaptic structure, the glomerular rosette (Hamori and Somogyi 1983). A combination of gain-of-function studies using mossy 
P.C. Salinas

fiber explants and analyses of Wnt7a mutant mice showed that Wnt7a, released by granule cells, acts on mossy fiber axons to promote the formation of complex glomerular rosettes and the accumulation of synaptic components to future presynaptic sites (Hall et al. 2000). Consistent with its role in presynaptic assembly, Wnt7a promotes synaptic vesicle recycling (Ahmad-Annuar et al. 2006; Cerpa et al. 2008). Together these findings show that Wnt7a promotes the assembly of synaptic boutons.

How does Wnt signaling regulate synaptic assembly? Different receptors mediate the synaptogenic activity of Wnts in hippocampal neurons (Davis et al. 2008; Varela-Nallar et al. 2009; Sahores et al. 2010). Gain of function of Fzl or Fz5 induces the clustering of synaptic vesicle proteins and active-zone proteins. In contrast, exposure to the soluble CRD domain of Fz1 or Fz5 or shRNA knockdown of Fz5 shows that these receptors are required for presynaptic assembly (Varela-Nallar et al. 2009; Sahores et al. 2010). Moreover, silencing of Fz5 blocks the ability of Wnt7a to promote presynaptic assembly, indicating that this receptor is required for Wnt7a function (Sahores et al. 2010). Other Wnt receptors also regulate the formation of synapses. For example, Wnt5a binds to and requires Ror tyrosine receptors to promote presynaptic assembly in cultured hippocampal neurons (Paganoni et al. 2010). Although the in vivo role of Frizzled and Ror receptors remains to be established, these studies show that in hippocampal cells three different receptors-Fz1, Fz5, and Ror-are involved in presynaptic assembly. These findings pose the question as to whether these molecules act as receptors for different Wnt ligands or whether they function in different subcellular compartments in hippocampal neurons.

Wnt signaling triggers a transcription-independent pathway to promote bouton assembly. In cultured hippocampal neurons, Wnt7a activates a signaling cascade that requires Dishevelled-1 (Dvl1) and inhibition of Gsk3ß for presynaptic assembly (Fig. 3). Dvll is present at

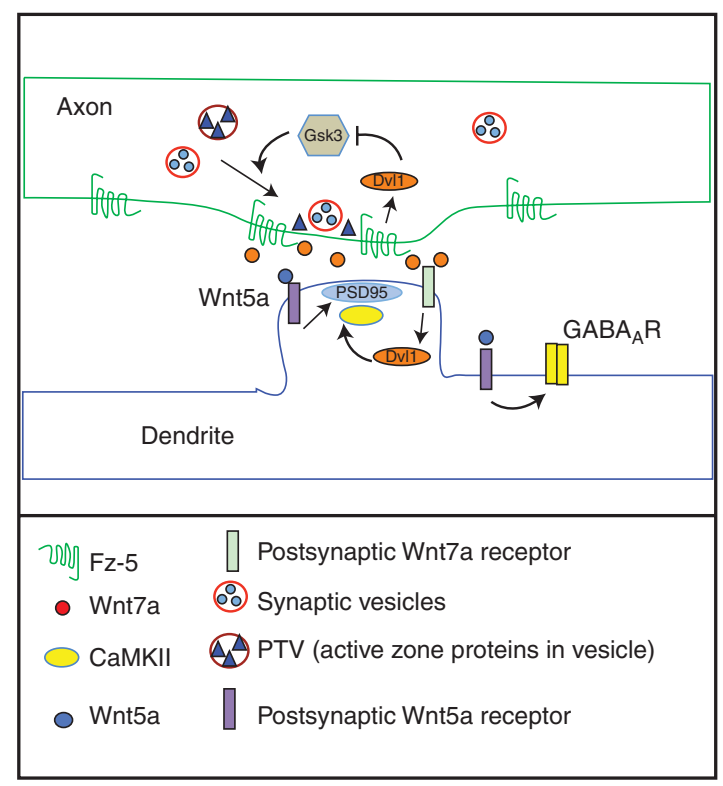

Figure 3. Wnts promote synapse formation. On axons, binding of Wnt7a to presynaptic Fz5 leads to Dvl activation, resulting in the inhibition of GSK3 $\beta$. This, in turn, promotes the recruitment of synaptic vesicles and active-zone proteins to future synaptic sites. On the postsynaptic dendrite, Wnt5a triggers the clustering of PSD-95 and promotes the formation of excitatory synapses. Wnt5a also increases the clustering of $\mathrm{GABA}_{\mathrm{A}} \mathrm{R}$ at inhibitory synapses. 
both sides of the synapse, suggesting that Wnt signaling might act presynaptically and postsynaptically (Ahmad-Annuar et al. 2006; Ciani et al.2011). In axons, gain of function of Dvl1 increases the number of neurotransmitter release sites and the clustering of presynaptic proteins. Conversely, axons from Dvl1 mutant neurons, which show fewer presynaptic sites, partially respond to exogenous Wnt7a, showing that Dvl1 is required by Wnt7a to promote presynaptic assembly (Ahmad-Annuar et al. 2006). The use of pharmacological inhibitors (Hall et al. 2000) and specific antagonists (Davis et al. 2008) of the canonical Wnt signaling pathway shows that axon remodeling and presynaptic assembly are achieved through inhibition of Gsk3. Similarly to the fly (Miech et al. 2008), blockade of transcription does not suppress the ability of Wnt7a to promote presynaptic assembly in cultured hippocampal neurons (EM Dickins and PC Salinas, unpubl.). How, then, do Wnts promote presynaptic assembly? A possible hypothesis is that Wnt signaling might promote the formation of synaptic boutons by modulating the microtubule cytoskeleton, resulting in changes in the transport of organelles or synaptic components to future synaptic sites. Future analyses using time-lapse recordings of fluorescently labeled synaptic molecules will provide important insights into the mechanism of Wnt-mediated synaptic assembly.

\section{Postsynaptic Assembly}

Studies at the Drosophila neuromuscular junction (NMJ) have clearly shown that Wg promotes the recruitment of both presynaptic and postsynaptic components (Packard et al. 2002). At vertebrate central synapses, Wnts also promote the recruitment of postsynaptic components. For example, Wnt5a increases the clustering of the postsynaptic scaffold protein PSD95 (Farias et al. 2009) and postsynaptic $\gamma$-aminobutyric acid type a $\left(\mathrm{GABA}_{\mathrm{A}}\right)$ receptors (Cuitino et al. 2010). Wnt7a or postsynaptic expression of Dvl1 increases the clustering of PSD95 and the colocalization of presynaptic and postsynaptic markers in hippocampal neurons (Ciani et al. 2011). Given that Wnt7a signals to the presyn- aptic axon through the Fz5 receptor, these studies show that Wnt7a signals bidirectionally to the axon and dendrite to promote synapse formation.

What is the mechanism responsible for postsynaptic assembly? Pharmacological studies have shown that Wnt5a promotes PSD95 clustering through JNK (Farias et al. 2009). In contrast, recruitment of $\mathrm{GABA}_{\mathrm{A}}$ receptors requires CaMKII activity (Cuitino et al. 2010). Although it is unclear whether Wnt5a directly regulates JNK and CaMKII on the postsynaptic side, Wnt5a rapidly increases the levels of $\mathrm{Ca}^{2+}$ in dendrites, suggesting that Wnt5a directly signals to the postsynaptic side (Varela-Nallar et al. 2010). Studies of Wnt7a and Dvl1 have shown that Wnt7a directly signals postsynaptically, because Dvl1 mutant dendrites fail to respond to Wnt7a. This postsynaptic effect of Wnt7a is mediated through the local activation CaMKII (Fig. 3) (Ciani et al. 2011). Thus, activation of CaMKII promotes both the assembly of postsynaptic structures of inhibitory and excitatory synapses. This poses the question of how activation of CaMKII can result in the assembly of two very different types of synapses. How is this achieved? Further studies will provide the answer.

\section{Excitatory versus Inhibitory Synapses}

In cultured hippocampal neurons, Wnt5a modulates inhibitory synapses by increasing the surface levels and the retention of $\mathrm{GABA}_{\mathrm{A}}$ receptors (Fig. 3) (Cuitino et al. 2010). Consistently, Wnt5a increases the amplitude of miniature inhibitory postsynaptic currents (mIPSCs). These results suggest that Wnt5a promotes the maturity of inhibitory synapses. Intriguingly, short exposure to Wnt5a does not affect the frequency of mIPSCs or the number of presynaptic sites (Cuitino et al. 2010). Time-course studies showed that Wnt5a first signals to the postsynaptic side to then induce presynaptic changes. Whether this effect is direct on the presynaptic axon remains to be determined.

Wnt5a also promotes the assembly of excitatory synapses as indicated by the increased clustering of PSD95, a marker of excitatory 
P.C. Salinas

postsynaptic sites (Farias et al. 2009). Although initial studies suggested that Wnt5a increases the formation of dendritic spines (Varela-Nallar et al. 2010), a recent publication suggests that Wnt5a does not affect spine formation (Cerpa et al. 2011). Recordings of fEPSCs showed that Wnt5a promotes glutamatergic transmission by increasing NMDA currents in hippocampal neurons. However, AMPA currents are unaffected (Cerpa et al. 2011). A Wnt/ $\mathrm{Ca}^{2+}$ signaling pathway regulates these postsynaptic changes as evident by the rapid increase in $\mathrm{Ca}^{2+}$ levels and the requirement of CaMKII activity (Varela-Nallar et al. 2010; Cerpa et al. 2011). Although loss-of-function approaches are needed to establish the in vivo role of Wnt5a at excitatory and inhibitory synapses, together these studies show that Wnt5a is a pansynaptogenic factor that promotes the assembly of both excitatory and inhibitory synapses.

In contrast to Wnt5a, Wnt7a specifically promotes the formation of excitatory synapses without affecting inhibitory ones (Ciani et al. 2011). Within 1 h, Wnt7a significantly increases the number of excitatory synapses and the frequency and amplitude of mEPSCs. However, the number of inhibitory sites and mIPSCs remains unchanged. In addition, Wnt7a increases the number and size of dendritic spines, postsynaptic structures that received excitatory inputs. In vivo analyses of the Wnt7a and Dvl1 double-mutant mice showed that Wnt7a-Dvl1 signaling is required for the formation and growth of spines at the CA 3 and CA 1 regions of the hippocampus. Cell-autonomous activation of the Wnt pathway in dendrites by expression of Dvl1 revealed that Wnt7a directly signals to dendrites to promote spine growth. Consistently, Dvl1 mutant dendrites do not respond to Wnt7a (Ciani et al. 2011). Interestingly, postsynaptic activation of Wnt signaling does not affect spine number, suggesting that this effect of Wnt7a is mediated through another mechanism. Analyses of the localization of Dvll and the use of a local reporter of CaMKII activity suggest that Wnt7a through postsynaptic Dvll locally activates CaMKII in dendritic spines (Ciani et al. 2011) to promote spine growth (Fig. 3). Thus, Wnt7a specifically promotes the formation of excitatory synapses by regulating dendritic spines.

Together, the studies on Wnt5a and Wnt7a suggest that Wnts differentially modulate distinct types of synapses. The specific effect of Wnt7a on excitatory synapses suggests that increased Wnt7a signaling could contribute to neurological disorders such as epilepsy. Future studies on Wnts will shed light into the mechanisms that control the balance between excitability and inhibition of neuronal circuits.

\section{NEURONAL ACTIVITY ON WNT RELEASE AND RECEPTOR LOCALIZATION}

Neuronal activity modulates neuronal circuit formation by regulating cell migration, axon guidance, dendritic development, and the formation and pruning of synapses (Budnik and Salinas 2011; West and Greenberg 2011). Several molecules including BDNF, neurotransmitter receptors, and adhesion molecules are regulated by activity (Meyer-Franke et al. 1998; Bozdagi et al. 2000; Du et al. 2000; Hayashi et al. 2000; Tanaka et al. 2000). However, little is known about the mechanisms by which neuronal activity sculptures neuronal networks. New studies show that Wnts and their receptors are under the control of neuronal activity and that they contribute to activity-dependent circuit formation and refinement.

The first indication that neuronal activity regulates Wnts came from studies in cultured hippocampal neurons. Depolarization with $\mathrm{KCl}$, which promotes dendritic development, increases Wnt activity in the media of cultured neurons, indicating that neural activity modulates the release, synthesis, or transcription of Wnts (Yu and Malenka 2003). Subsequent studies revealed that activation of NMDA receptors increases the transcription of Wnt2, a protein expressed in the hippocampus. Importantly, blockade of Wnt2 compromised dendritic development (Wayman et al. 2006). Further evidence comes from studies showing that the levels of $\mathrm{Wnt} 7 \mathrm{a} / \mathrm{b}$ protein increase in the hippocampus of animals exposed to an enriched environment (Gogolla et al. 2009).

Changes in neuronal activity also influence the localization and trafficking of Frizzled re- 
Wnt Signaling in the Vertebrate CNS

ceptors. High-frequency stimulation, which induces synaptic potentiation, increases the levels of surface Fz5 (sFz5) without affecting the total level of the receptor in hippocampal neurons. Conversely, low-frequency stimulation (LFS), which elicits long-term depression, decreases the levels of sFz5. Importantly, HFS increases, whereas LFS decreases the amount of sFz5 receptor at synapses and the percentage of synapses containing Fz5 (Sahores et al. 2010). Given that $\mathrm{Fz}$ localization is regulated by Wnt ligands (Mathew et al. 2005; Klassen and Shen 2007; Mosca and Schwarz 2010) and that activity promotes the expression of Wnts (Wayman et al. 2006; Korkut et al. 2009), the contribution of Wnts in Fz5 localization was investigated. Wnt blockade with the secreted Wnt antagonist Sfrps or with the extracellular domain of Fz5 showed that endogenous Wnts expressed by hippocampal neurons mediate the effect of neuronal activity on Fz5 localization. Thus, patterned ac- tivity induced by HFS regulates the release or expression of Wnts, which then promote the trafficking of Fz5 to the cell surface and to the synapse (Fig. 4). Future studies focused on the mechanisms that control the trafficking of $\mathrm{Fz}$ receptors to synapses will provide important insights into the effect of ligands on their receptor localization and also how neuronal activity modulates synapse formation and function.

\section{STRUCTURAL AND FUNCTIONAL PLASTICITY OF SYNAPSES}

The expression of Wnts, their receptors, and several components of the Wnt pathway in the mature brain suggests that Wnts could also regulate synaptic plasticity in the adult. The first hint that synaptic function is modulated by Wnt signaling came from analyses of $W n t 7 a$; Dvl1 double-mutant mice. In these mice, the complexity of the glomerular rosettes is affected,
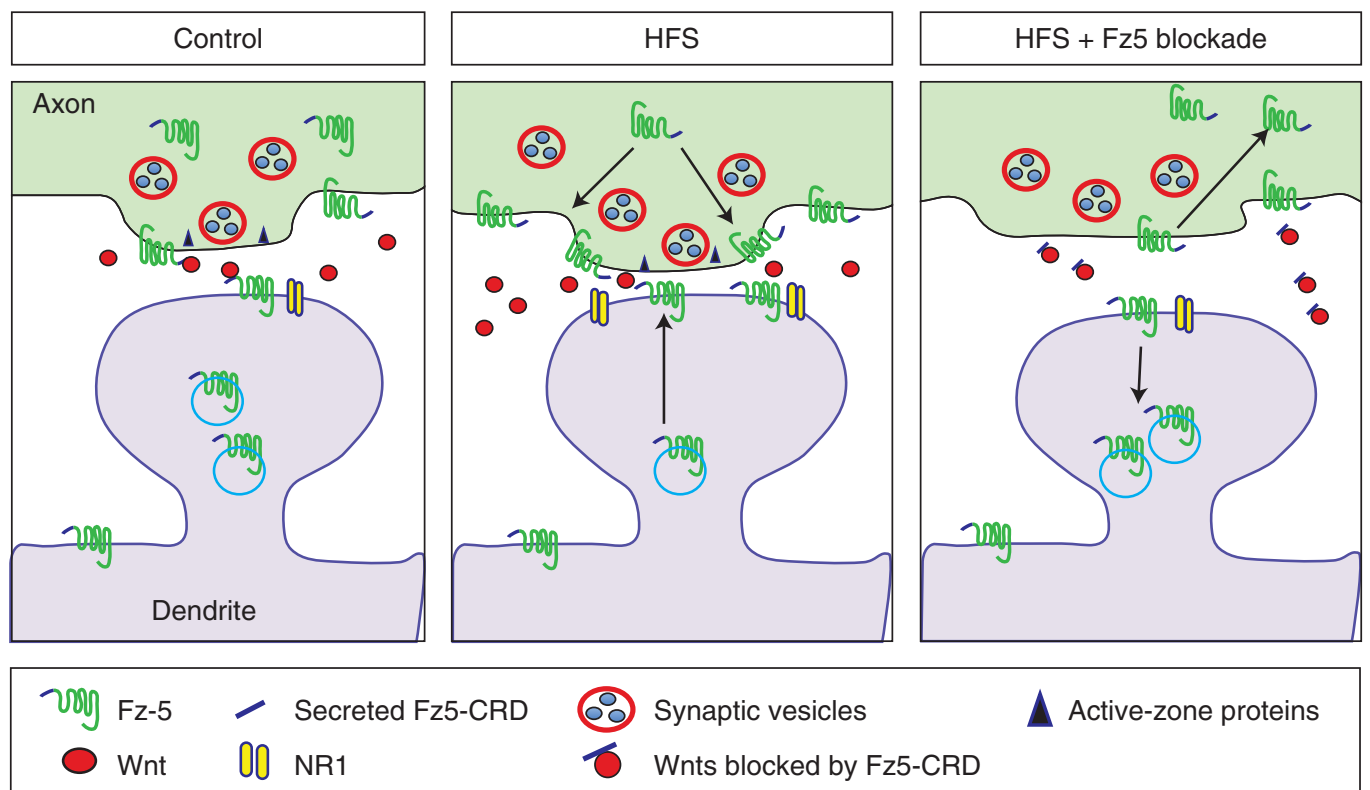

Synaptic vesicles

Active-zone proteins

Figure 4. Neuronal activity regulates the membrane insertion of Fz5 receptors and their localization to synapses. In hippocampal neurons, surface Fz5 is localized to synaptic and extrasynaptic sites under basal conditions. Increased neuronal firing induced by high-frequency stimulation (HFS) increases the mobilization of Fz5 to the cell surface and in particular to the surface of synapses. Blockade of endogenous Wnt factors with the soluble extracellular domain of Fz5 (CRD-Fz5) suppresses the effect of HFS on Fz5 localization. Importantly, CRD-Fz5 completely blocks activity-induced synapse formation as indicated by the decrease in the colocalization of presynaptic vesicle markers and NMDARs. 
P.C. Salinas

but the presynaptic active zones appear normal. Interestingly, electrophysiological recordings showed defects in the frequency of mEPSCs, suggesting a potential defect in neurotransmitter release (Ahmad-Annuar et al. 2006). A more recent study has reinforced this hypothesis. Electrophysiological recording of brain slices showed that Wnt7a decreases pair pulse facilitation at the hippocampal CA3-CA1 synapse, suggesting a role for Wnt7a in the regulation of neurotransmitter release (Cerpa et al. 2008). This process could be regulated at different levels of the synaptic vesicle cycle, from changes in the reserved pool of vesicles to the rate of docking, fusion, or endocytosis. Dynamics studies of synaptic vesicles labeled with the lipophilic dye FM-143 suggest that Wnt7a increases synaptic vesicle exocytosis (Cerpa et al. 2008). However, further detailed characterization is required to fully establish a role for Wnt signaling in neurotransmitter release.

Wnt signaling regulates synaptic strength through postsynaptic changes. As discussed before, analyses of Wnt signaling-deficient mutant mice revealed that Wnt7a signaling is essential for spine formation and growth in the hippocampus. Increased spine growth has been correlated with an increase in synaptic strength (Yuste and Bonhoeffer 2001; Matsuzaki et al. 2004; Bourne and Harris 2008). Indeed, Wnt7a/ Dvl1 double-mutant mice show deficits in synaptic strength in the hippocampus. Conversely, gain-of-function studies with exogenous Wnt7a or expression of Dvll in dendrites show that Wnt7a-Dvll signaling increases synaptic strength at excitatory synapses in a CaMKII-dependent manner (Ciani et al. 2011). Thus, Wnt7a signaling modulates the structural and synaptic plasticity of neuronal circuits in the hippocampus.

Several studies suggest that Wnt signaling also modulates long-term potentiation. In acute brain slices, blockade of Wnt signaling with specific antibodies against Wnt3a or the secreted extracellular domain of Frizzled-8 weakly decreases long-term potentiation (Chen et al. 2006). Conversely, activation of the canonical Wnt signaling with lithium or with Wnt3a mildly increases potentiation. A more recent study suggests that
Wnt5a, through a non-canonical Wnt pathway, modulates the induction of synaptic potentiation at the CA3-CA1 synapse (Cerpa et al. 2011). Thus, Wnts, through the activation of canonical or non-canonical pathways, regulate long-term potentiation. Further studies are essential to elucidate the contribution of Wnt signaling to maintenance of long-term synaptic plasticity.

Wnt signaling also contributes to the structural plasticity of synaptic structures in the central nervous system. In the hippocampus, mossy fiber axons form complex terminal protrusions with dendritic spines of the CA3 region (Rollenhagen et al. 2007). When mice are exposed to an enriched environment (EE), these structures become extensively remodeled as mossy fiber terminals interdigitate extensively with CA3 dendrites (Gogolla et al. 2009). Because this modeling is similar to that observed by Wnt7a in the cerebellar mossy fibers (Hall et al. 2000), the role of Wnts was investigated. Three pieces of data support the role of Wnt7a in EE-mediated remodeling. First, Wnt7a protein levels are elevated in the hippocampus of EE animals. Second, blockade of Wnt7a by injection of the Wnt antagonist Sfrp1 suppresses EE-mediated axon remodeling. Third, injection of exogenous Wnt7a into the hippocampus mimics the effect of EE (Gogolla et al. 2009). These studies raise the interesting possibility that Wnts might also modulate structural changes following sensory experience. Indeed, Wnt signaling mediates visual experience-dependent plasticity. In the optic tectum, the receptive field (RF), which depends on the number of retinal ganglia cell axons, decreases in size during development in an activity-dependent manner. Acute blockade of Wnts or infusion of Wnts in the Xenopus tectum interferes with this developmental reduction in RF (Lim et al. 2010), suggesting that Wnt signaling regulates the refinement of neuronal connections during sensory experience-dependent plasticity.

Activity-mediated synapse formation is also modulated by Wnt proteins through the $\mathrm{Fz}-5$ receptor. As mentioned above, HFS increases the level of Fz5 receptor at the surface and at synapses (Sahores et al. 2010). This increased level of Fz5 at synapses is correlated with the formation and 
Wnt Signaling in the Vertebrate CNS

growth of synapses. Importantly, blockade of endogenous Wnts with Sfrps or with the soluble extracellular domain of the receptor Fz5 completely suppresses the effect of HFS on synapse formation. Thus, Wnt signaling through Fz5 significantly contributes to activity-mediated synapse formation in hippocampal neurons.

\section{NEUROLOGICAL DISORDERS AND NEURODEGENERATION}

Mounting evidence suggests a link between dysfunction of Wnt signaling and neurological disorders as well as neurodegenerative diseases. Mutations in the disrupted in schizophrenia (DISC1) gene were found in a Scottish family with a high incidence of schizophrenia, major depression, and bipolar disorder (Porteous and Millar 2006). Two recent studies showed that DISC1 modulates canonical Wnt signaling. DISC1 interacts with and inhibits Gsk3 $\beta$ activity, resulting in increased $\beta$-catenin signaling (Mao et al. 2009). Moreover, DISC1 also interacts with the scaffold protein DIX domain-containing protein (DIXDC1), which together with DSC1 modulates canonical Wnt signaling (Singh et al. 2010). In addition, Gsk3 inhibitors restore the behavioral defects manifested in DISC1-deficient mutant mice (Mao et al. 2009). Because different signaling pathways regulate Gsk3 activity, further studies are needed to establish whether deficiency in Wnt signaling contributes to schizophrenia.

Deregulation of Wnt signaling might contribute to the changes in mood and behavior characteristic of psychiatric disorders such as manic depression. The first evidence that Wnt signaling contributes to social behavior came from analyses of Dvl1 mutant mice, which show defects in social interactions and deficits in the acoustic startled reflex (Lijam et al. 1997). Further support came from the finding that lithium, used for the treatment of bipolar affective disorders (O'Brien and Klein 2007), is a direct inhibitor of Gsk3 (Klein and Melton 1996; Stambolic et al. 1996). Although many molecular targets of lithium have been identified, the mechanism by which lithium modulates mood is currently unclear. Nevertheless, Gsk3 remains a strong candi- date because heterozygous Gsk3 mutant mice show a similar phenotype to mice treated with lithium (Beaulieu et al. 2004; O'Brien et al. 2004). These findings open the doors for developing new therapeutic strategies for the treatment of mood disorders.

In the aging brain, deficiency in canonical Wnt signaling has been associated with $\mathrm{Alz}$ heimer's disease (AD). The link between increased Gsk3 activity and AD has been known (Hernandez et al. 2010). Yet only recently, a genetic link between decreased Wnt signaling and $\mathrm{AD}$ has been presented. Genome-wide studies have identified a broad susceptibility region for later-onset Alzheimer's disease in chromosome 12, which contains the LRP6 locus (D'Introno et al. 2006). Importantly, a common variant of LRP6, which shows decreased levels of canonical Wnt signaling in heterologous cells, has been associated with late-onset AD (De Ferrari et al. 2007). Further evidence comes from the finding that Dickkopf-1 (Dkk1), the secreted Wnt antagonist that specifically blocks canonical Wnt signaling through binding to LRP6, is elevated in $\mathrm{AD}$ brain biopsies and in the brain of transgenic mouse models for AD (Caricasole et al. 2004; Rosi et al. 2010). Together, these findings suggest that decreased canonical Wnt signaling might contribute to $\mathrm{AD}$ pathogenesis. Future studies are essential to determine a role of Wnt signaling in this devastating neurodegenerative disease.

\section{CONCLUSIONS}

In the last few years, great progress has been made in understanding the role of Wnt signaling in the formation of neuronal circuits during development. It is now evident that Wnts also regulate different aspects of synaptic plasticity, through presynaptic and postsynaptic mechanisms. Moreover, Wnts mediate activity-dependent processes such as synapse formation, synaptic remodeling, and circuit refinement. Finally, there is increased evidence suggesting a potential link between dysfunction of Wnt signaling and developmental disorders and bipolar affective disorders. Future studies on Wnts in the developing, mature, and aging brain will provide 
P.C. Salinas

crucial information for developing therapeutic approaches for the treatment and/or prevention of neurological disorders and neurodegeneration.

\section{ACKNOWLEDGMENTS}

I thank members of my laboratory for their useful and insightful comments on the manuscript. I also thank the MRC, Wellcome Trust, BBSRC, ARUK, and EU for supporting our work.

\section{REFERENCES}

Ahmad-Annuar A, Ciani L, Simeonidis I, Herreros J, Fred NB, Rosso SB, Hall A, Brickley S, Salinas PC. 2006. Signaling across the synapse: A role for Wnt and Dishevelled in presynaptic assembly and neurotransmitter release. $J$ Cell Biol 174: 127-139.

Beaulieu JM, Sotnikova TD, Yao WD, Kockeritz L, Woodgett JR, Gainetdinov RR, Caron MG. 2004. Lithium antagonizes dopamine-dependent behaviors mediated by an AKT/glycogen synthase kinase 3 signaling cascade. Proc Natl Acad Sci 101: 5099-5104.

Bourne JN, Harris KM. 2008. Balancing structure and function at hippocampal dendritic spines. Ann Rev Neurosci 31: 47-67.

Bozdagi O, Shan W, Tanaka H, Benson DL, Huntley GW. 2000. Increasing numbers of synaptic puncta during latephase LTP: N-Cadherin is synthesized, recruited to synaptic sites, and required for potentiation. Neuron $\mathbf{2 8}$ 245-259.

Budnik V, Salinas PC. 2011. Wnt signaling during synaptic development and plasticity. Curr Opin Neurobiol 21: 151-159.

Caricasole A, Copani A, Caraci F, Aronica E, Rozemuller AJ, Caruso A, Storto M, Gaviraghi G, Terstappen GC, Nicoletti F. 2004. Induction of Dickkopf-1, a negative modulator of the Wnt pathway, is associated with neuronal degeneration in Alzheimer's brain. J Neurosci 24: 60216027.

Cerpa W, Godoy JA, Alfaro I, Farias GG, Metcalfe MJ, Fuentealba R, Bonansco C, Inestrosa NC. 2008. Wnt-7a modulates the synaptic vesicle cycle and synaptic transmission in hippocampal neurons. J Biol Chem 283: 5918 5927.

Cerpa M, Gambrill A, Inestrosa N, Barria A. 2011. Regulation of NMDA-receptor synaptic transmission by Wnt signaling. J Neurosci 31: 9466-9471.

Chen J, Park CS, Tang SJ. 2006. Activity-dependent synaptic Wnt release regulates hippocampal long term potentiation. J Biol Chem 281: 11910-11916.

Ciani L, Krylova O, Smalley MJ, Dale TC, Salinas PC. 2004. A divergent canonical WNT-signaling pathway regulates microtubule dynamics: Dishevelled signals locally to stabilize microtubules. J Cell Biol 164: 243-253.

Ciani L, Boyle K, Dickins E, Sahores M, Anane D, Lopes DM, Gibb A, Salinas PC. 2011. Wnt7a signaling pro- motes dendritic spine growth and synaptic strength through $\mathrm{Ca}^{2+} /$ calmodulin-dependent protein kinase II. Proc Natl Acad Sci 108: 10732-10737.

Cuitino L, Godoy JA, Farias GG, Couve A, Bonansco C, Fuenzalida M, Inestrosa NC. 2010. Wnt-5a modulates recycling of functional $\mathrm{GABA}_{\mathrm{A}}$ receptors on hippocampal neurons. J Neurosci 30: 8411-8420.

Davis EK, Zou Y, Ghosh A. 2008. Wnts acting through canonical and noncanonical signaling pathways exert opposite effects on hippocampal synapse formation. Neural Dev 3: 32.

De Ferrari GV, Papassotiropoulos A, Biechele T, Wavrant De-Vrieze F, Avila ME, Major MB, Myers A, Saez K, Henriquez JP, Zhao A, et al. 2007. Common genetic variation within the low-density lipoprotein receptor-related protein 6 and late-onset Alzheimer's disease. Proc Natl Acad Sci 104: 9434-9439.

D’Introno A, Solfrizzi V, Colacicco AM, Capurso C, Amodio M, Todarello O, Capurso A, Kehoe PG, Panza F. 2006. Current knowledge of chromosome 12 susceptibility genes for late-onset Alzheimer's disease. Neurobiol Aging 27: 1537-1553.

Du J, Feng L, Yang F, Lu B. 2000. Activity- and $\mathrm{Ca}^{2+}$ dependent modulation of surface expression of brainderived neurotrophic factor receptors in hippocampal neurons. J Cell Biol 150: 1423-1434.

Dunaevsky A, Mason CA. 2003. Spine motility: A means towards an end? Trends Neurosci 26: 155-160.

Farias GG, Alfaro IE, Cerpa W, Grabowski CP, Godoy JA, Bonansco C, Inestrosa NC. 2009. Wnt-5a/JNK signaling promotes the clustering of PSD-95 in hippocampal neurons. J Biol Chem 284: 15857-15866.

Fradkin LG, Noordermeer JN, Nusse R. 1995. The Drosophila Wnt protein DWnt-3 is a secreted glycoprotein localized on the axon tracts of the embryonic CNS. Dev Biol 168: $202-213$.

Fradkin LG, Dura JM, Noordermeer JN. 2010. Ryks: New partners for Wnts in the developing and regenerating nervous system. Trends Neurosci 33: 84-92.

Gogolla N, Galimberti I, Deguchi Y, Caroni P. 2009. Wnt signaling mediates experience-related regulation of synapse numbers and mossy fiber connectivities in the adult hippocampus. Neuron 62: 510-525.

Hall AC, Lucas FR, Salinas PC. 2000. Axonal remodeling and synaptic differentiation in the cerebellum is regulated by WNT-7a signaling. Cell 100: 525-535.

Hamori J, Somogyi J. 1983. Differentiation of cerebellar mossy fiber synapses in the rat: A quantitative electron microscope study. J Comp Neurol 220: 365-377.

Hayashi Y, Shi SH, Esteban JA, Piccini A, Poncer JC, Malinow R. 2000. Driving AMPA receptors into synapses by LTP and CaMKII: Requirement for GluR1 and PDZ domain interaction. Science 287: 2262-2267.

Hernandez F, Gomez de Barreda E, Fuster-Matanzo A, Lucas JJ, Avila J. 2010. GSK3: A possible link between $\beta$ amyloid peptide and tau protein. Exp Neurol 223: 322-325.

Hindges R, McLaughlin T, Genoud N, Henkemeyer M, O'Leary DD. 2002. EphB forward signaling controls directional branch extension and arborization required for dorsal-ventral retinotopic mapping. Neuron 35: $475-487$. 
Hutchins BI, Li L, Kalil K. 2011. Wnt/calcium signaling mediates axon growth and guidance in the developing corpus callosum. Dev Neurobiol 71: 269-283.

Imondi R, Thomas JB. 2003. Neuroscience. The ups and downs of Wnt signaling. Science 302: 1903-1904.

Keeble TR, Halford MM, Seaman C, Kee N, Macheda M, Anderson RB, Stacker SA, Cooper HM. 2006. The Wnt receptor Ryk is required for Wnt5a-mediated axon guidance on the contralateral side of the corpus callosum. $J$ Neurosci 26: $5840-5848$.

Klassen MP, Shen K. 2007. Wnt signaling positions neuromuscular connectivity by inhibiting synapse formation in C. elegans. Cell 130: 704-716.

Klein PS, Melton DA. 1996. A molecular mechanism for the effect of lithium on development. Proc Natl Acad Sci 93: 8455-8459.

Korkut C, Ataman B, Ramachandran P, Ashley J, Barria R, Gherbesi N, Budnik V. 2009. Trans-synaptic transmission of vesicular Wnt signals through Evi/Wntless. Cell 139: 393-404.

Krylova O, Herreros J, Cleverley KE, Ehler E, Henriquez JP, Hughes SM, Salinas PC. 2002. WNT-3, expressed by motoneurons, regulates terminal arborization of neurotrophin-3-responsive spinal sensory neurons. Neuron 35: 1043-1056.

Li L, Hutchins BI, Kalil K. 2009. Wnt5a induces simultaneous cortical axon outgrowth and repulsive axon guidance through distinct signaling mechanisms. J Neurosci 29: 5873-5883.

Lijam N, Paylor R, McDonald MP, Crawley JN, Deng CX, Herrup K, Stevens KE, Maccaferri G, McBain CJ, Sussman DJ, et al. 1997. Social interaction and sensorimotor gating abnormalities in mice lacking Dvl1. Cell 90: $895-$ 905.

Lim BK, Cho SJ, Sumbre G, Poo MM. 2010. Region-specific contribution of ephrin-B and Wnt signaling to receptive field plasticity in developing optic tectum. Neuron 65: 899-911.

Liu Y, Shi J, Lu CC, Wang ZB, Lyuksyutova AI, Song XJ, Zou Y. 2005. Ryk-mediated Wnt repulsion regulates posterior-directed growth of corticospinal tract. Nat Neurosci 8: $1151-1159$.

Luo L. 2006. Developmental neuroscience: Two gradients are better than one. Nature 439: 23-24.

Lyuksyutova AI, Lu CC, Milanesio N, King LA, Guo N, Wang Y, Nathans J, Tessier-Lavigne M, Zou Y. 2003. Anterior-posterior guidance of commissural axons by Wntfrizzled signaling. Science 302: 1984-1988.

Mao Y, Ge X, Frank CL, Madison JM, Koehler AN, Doud MK, Tassa C, Berry EM, Soda T, Singh KK, et al. 2009. Disrupted in schizophrenia 1 regulates neuronal progenitor proliferation via modulation of GSK3 $\beta / \beta$-catenin signaling. Cell 136: 1017-1031.

Mathew D, Ataman B, Chen J, Zhang Y, Cumberledge S, Budnik V. 2005. Wingless signaling at synapses is through cleavage and nuclear import of receptor DFrizzled2. Science 310: 1344-1347.

Matsuzaki M, Honkura N, Ellis-Davies GC, Kasai H. 2004. Structural basis of long-term potentiation in single dendritic spines. Nature 429: 761-766.
McLaughlin T, O’Leary DD. 2005. Molecular gradients and development of retinotopic maps. Annu Rev Neurosci 28: 327-355.

Meyer-Franke A, Wilkinson GA, Kruttgen A, Hu M, Munro E, Hanson MG Jr, Reichardt LF, Barres BA. 1998. Depolarization and cAMP elevation rapidly recruit TrkB to the plasma membrane of CNS neurons. Neuron 21: 681-693.

Miech C, Pauer HU, He X, Schwarz TL. 2008. Presynaptic local signaling by a canonical wingless pathway regulates development of the Drosophila neuromuscular junction. J Neurosci 28: 10875-10884.

Mosca TJ, Schwarz TL. 2010. The nuclear import of Frizzled2-C by Importins- $\beta 11$ and $\alpha 2$ promotes postsynaptic development. Nat Neurosci 13, 935-943.

O’Brien WT, Klein PS. 2007. Regulation of glycogen synthase kinase-3 in patients with affective disorders. Biol Psychiatry 61: 139-141.

O’Brien WT, Harper AD, Jove F, Woodgett JR, Maretto S, Piccolo S, Klein PS. 2004. Glycogen synthase kinase-3 $\beta$ haploinsufficiency mimics the behavioral and molecular effects of lithium. J Neurosci 24: 6791-6798.

Okabe S, Miwa A, Okado H. 2001. Spine formation and correlated assembly of presynaptic and postsynaptic molecules. J Neurosci 21: 6105-6114.

Packard M, Koo ES, Gorczyca M, Sharpe J, Cumberledge S, Budnik V. 2002. The Drosophila Wnt, wingless, provides an essential signal for pre- and postsynaptic differentiation. Cell 111: 319-330.

Paganoni S, Bernstein J, Ferreira A. 2010. Ror1-Ror2 complexes modulate synapse formation in hippocampal neurons. Neuroscience 165: 1261-1274.

Porteous DJ, Millar JK. 2006. Disrupted in schizophrenia 1: Building brains and memories. Trends Mol Med 12: 255-261.

Purro SA, Ciani L, Hoyos-Flight M, Stamatakou E, Siomou E, Salinas PC. 2008. Wnt regulates axon behavior through changes in microtubule growth directionality: A new role for adenomatous polyposis coli. J Neurosci 28: 8644-8654.

Rollenhagen A, Satzler K, Rodriguez EP, Jonas P, Frotscher M, Lubke JH. 2007. Structural determinants of transmission at large hippocampal mossy fiber synapses. J Neurosci 27: 10434-10444.

Rosi MC, Luccarini I, Grossi C, Fiorentini A, Spillantini MG, Prisco A, Scali C, Gianfriddo M, Caricasole A, Terstappen GC, et al. 2010. Increased Dickkopf-1 expression in transgenic mouse models of neurodegenerative disease. J Neurochem 112: 1539-1551.

Sahores M, Gibb A, Salinas PC. 2010. Frizzled-5, a receptor for the synaptic organizer Wnt7a, regulates activitymediated synaptogenesis. Development 137: 2215-2225.

Salinas PC, Zou Y. 2008. Wnt signaling in neural circuit assembly. Annu Rev Neurosci 31: 339-358.

Sanchez-Camacho C, Bovolenta P. 2009. Emerging mechanisms in morphogen-mediated axon guidance. Bioessays 31: $1013-1025$.

Schmitt AM, Shi J, Wolf AM, Lu CC, King LA, Zou Y. 2006. Wnt-Ryk signalling mediates medial-lateral retinotectal topographic mapping. Nature 439: 31-37. 
P.C. Salinas

Shafer B, Onishi K, Lo C, Colakoglu G, Zou Y. 2011. Vangl2 promotes Wnt/planar cell polarity-like signaling by antagonizing Dvl1-mediated feedback inhibition in growth cone guidance. Dev Cell 20: 177-191.

Singh KK, Ge X, Mao Y, Drane L, Meletis K, Samuels BA Tsai LH. 2010. Dixdc1 is a critical regulator of DISC1 and embryonic cortical development. Neuron 67: 33-48.

Stambolic V, Ruel L, Woodgett JR. 1996. Lithium inhibits glycogen synthase kinase-3 activity and mimics wingless signalling in intact cells. Curr Biol 6: 1664-1668.

Tanaka H, Shan W, Phillips GR, Arndt K, Bozdagi O, Shapiro L, Huntley GW, Benson DL, Colman DR. 2000. Molecular modification of $N$-cadherin in response to synaptic activity. Neuron 25: 93-107.

Trachtenberg JT, Chen BE, Knott GW, Feng G, Sanes JR, Welker E, Svoboda K. 2002. Long-term in vivo imaging of experience-dependent synaptic plasticity in adult cortex. Nature 420: 788-794.

Varela-Nallar L, Grabowski CP, Alfaro IE, Alvarez AR, Inestrosa NC. 2009. Role of the Wnt receptor Frizzled-1 in presynaptic differentiation and function. Neural Dev 4: 41.
Varela-Nallar L, Alfaro IE, Serrano FG, Parodi J, Inestrosa NC. 2010. Wingless-type family member 5A (Wnt-5a) stimulates synaptic differentiation and function of glutamatergic synapses. Proc Natl Acad Sci 107: 21164-21169.

Wayman GA, Impey S, Marks D, Saneyoshi T, Grant WF, Derkach V, Soderling TR. 2006. Activity-dependent dendritic arborization mediated by CaM-kinase I activation and enhanced CREB-dependent transcription of Wnt-2. Neuron 50: 897-909.

West AE, Greenberg ME. 2011. Neuronal activity-regulated gene transcription in synapse development and cognitive function. Cold Spring Harb Perspect Biol doi: 10.1101/ cshperspect.a005744.

Yoshihara Y, De Roo M, Muller D. 2009. Dendritic spine formation and stabilization. Curr Opin Neurobiol 19: 146153.

Yu X, Malenka RC. 2003. $\beta$-Catenin is critical for dendritic morphogenesis. Nat Neurosci 6: 1169-1177.

Yuste R, Bonhoeffer T. 2001. Morphological changes in dendritic spines associated with long-term synaptic plasticity. Annu Rev Neurosci 24: 1071-1089. 


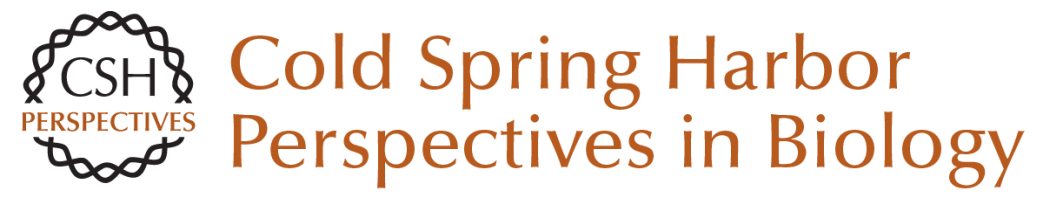

\section{Wnt Signaling in the Vertebrate Central Nervous System: From Axon Guidance to Synaptic Function}

Patricia C. Salinas

Cold Spring Harb Perspect Biol 2012; doi: 10.1101/cshperspect.a008003

Subject Collection Wnt Signaling

Wnt Signaling in Vertebrate Axis Specification

Hiroki Hikasa and Sergei Y. Sokol

Secreted and Transmembrane Wnt Inhibitors and

Activators

Cristina-Maria Cruciat and Christof Niehrs

Wnt Signaling in Normal and Malignant

Hematopoiesis

William Lento, Kendra Congdon, Carlijn Voermans, et al.

Frizzled and LRP5/6 Receptors for Wnt/ $\beta$-Catenin Signaling

Bryan T. MacDonald and Xi He

TCF/LEFs and Wnt Signaling in the Nucleus

Ken M. Cadigan and Marian L. Waterman

\section{Alternative Wnt Pathways and Receptors \\ Renée van Amerongen}

ß-Catenin-Dependent Wnt Signaling in C. elegans:

Teaching an Old Dog a New Trick

Belinda M. Jackson and David M. Eisenmann

The Evolution of the Wnt Pathway

Thomas W. Holstein

The $\beta$-Catenin Destruction Complex

Jennifer L. Stamos and William I. Weis

Wnt Signaling in Skin Development, Homeostasis, and Disease Xinhong Lim and Roel Nusse

Wnt Signaling in Bone Development and Disease:

Making Stronger Bone with Wnts Jean B. Regard, Zhendong Zhong, Bart O.

Williams, et al.

Targeting Wnt Pathways in Disease Zachary F. Zimmerman, Randall T. Moon and Andy J. Chien

Wnt Signaling in Mammary Glands: Plastic Cell

Fates and Combinatorial Signaling Caroline M. Alexander, Shruti Goel, Saja A. Fakhraldeen, et al.

Wnt Signaling and Injury Repair Jemima L. Whyte, Andrew A. Smith and Jill A. Helms

Wnt Signaling and Forebrain Development Susan J. Harrison-Uy and Samuel J. Pleasure

Wnt Signaling in Neuromuscular Junction Development Kate Koles and Vivian Budnik

For additional articles in this collection, see http://cshperspectives.cshlp.org/cgi/collection/

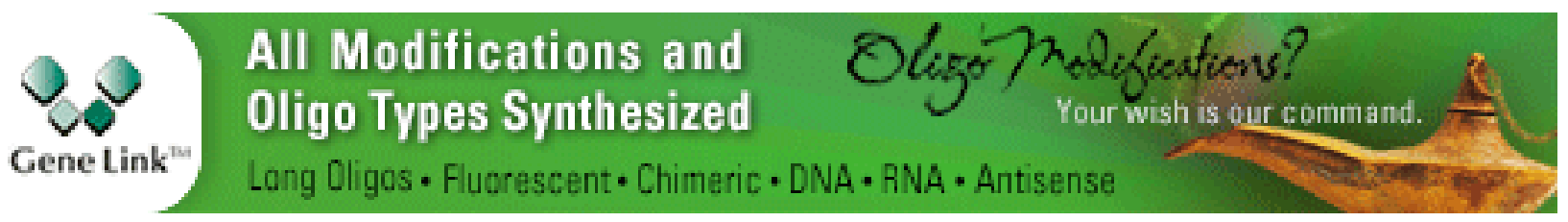

